\title{
Angiomyolipoma mimicking hepatocellular carcinoma on preoperative magnetic resonance image: The way to prevent unnecessary surgery for hepatic angiomyolipoma
}

\author{
Hyeo Seong HWANG ${ }^{1}$, Young Nyun PARK ${ }^{2}$, Dai Hoon HAN ${ }^{* 1}$, Gi Hong CHOI', Kyung Sik KIM', Jin Sub CHOI' \\ 'Department of Surgery, Yonsei University College of Medicine, Seoul, Korea \\ ${ }^{2}$ Department of Pathology, Yonsei University College of Medicine, Seoul, Korea
}

Introduction: The magnetic resonance imaging (MRI) of hepatic angiomyolipoma (AML) is often mistaken for hepatocellular carcinoma (HCC). In this study, clinicopathological, and imaging features of hepatic AML misdiagnosed with a malignant tumor before surgery were reviewed.

Methods: A total of 34 patients diagnosed with hepatic AML by surgery $(\mathrm{n}=20)$ or biopsy $(\mathrm{n}=14)$ were included (2008-2020). Twenty female and fourteen male patients with a mean age of 52.9 years (range 28-78) were followed-up for about 68.23 months.

Results: Only one patient was positive for HBsAg among 34 cases, and preoperative levels of serum alpha-fetoprotein and protein induced by vitamin K absence-II were not increased for all patients. Two patients showed non-alcoholic fatty liver disease. Twenty-nine patients have solitary liver tumor and the remainder has multiple ones. In two cases, renal AML was simultaneously diagnosed. In preoperative MRI, AML was misdiagnosed as HCC $(\mathrm{n}=14,41.2 \%)$, adenoma $(\mathrm{n}=1,2.9 \%)$, cholangiocellular carcinoma $(\mathrm{n}=1,2.9 \%)$, intrahepatic metastasis of malignant tumor $(\mathrm{n}=2,5.8 \%)$, or inconclusive opinions $(\mathrm{n}=6,17.6 \%)$. In subgroup analysis, overall survival was not different $(p=0.496)$, on the contrary, biopsy group showed significantly short length of hospitalization $(p=0.001)$, small tumor size $(23.5 \mathrm{~mm}$ versus $45.8 \mathrm{~mm}, p=0.017)$, better diagnostic accuracy (50\% versus $15.0 \%, p=0.011)$. In immunohistochemical analysis, several antibodies were detected including HMB45 (in 32 cases), SMA ( $n=19)$, CD 34 ( $n=7$ ), Melanin A ( $=5$ ).

Conclusions: Since AML is a benign tumor, for the suspicious HCC suddenly discovered in a patient without clinical risk factors, it is better to perform biopsy first to prevent unnecessary surgery. 